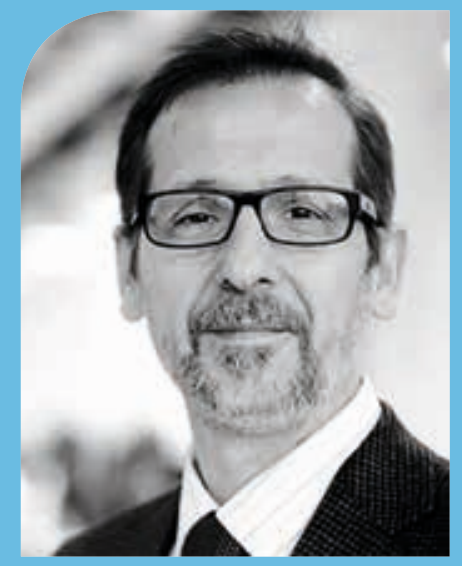

ANDREA SUMMER

Presidente AITeL

\title{
Lo possiamo raccontare ill nostro latte?
}

\section{Can we talk about our milk?}

\section{Cari Soci AlTeL e Cari Lettori,}

abbiamo già parlato diverse volte dei consumi di latte che calano, delle fake news con al centro il nostro mondo lattiero caseario. È un problema non solo italiano. Correre dietro ad ogni singola falsa affermazione che getta discredito sul latte è praticamente impossibile.

Proviamo, invece, a fare noi una comunicazione attiva. Chiediamoci allora, in tutta onestà e trasparenza, se possiamo, se ce la sentiamo, di raccontare la storia del nostro latte- tha sua originetasuastoria.

La nostra è una filiera unitaria. Si parte dall'allevamento per arrivare alla distribuzione del prodotto. Non possiamo segmentarla. Non possiamo tralasciarne un pezzo.

Dobbiamo porre attenzione alle giuste richieste dei nostri consumatori. I quali ci chiedono sempre più, una forte attenzione all'ambiente ed al benessere animale, come già ampiamente detto.

Dobbiamo pensare ad allevamenti che si interrogano sulla loro sostenibilità. Sostenibilità economica, sociale e ambientale. Dobbiamo orientare i nostri allevamenti ad avere la massima attenzione per l'impatto che hanno sull'ambiente. Studiare e applicare le modalità per una produzione che sia il meno impattante possibile.

Tutto questo senza perdere di vista il benessere animale, premiandolo e migliorandolo. Aspetto che sicuramente porta a dei vantaggi importanti, oltre ad essere eticamente essenziale. Infatti, animali che stanno bene producono meglio e di più.

Sappiamo che il nostro allevamento fa parte di quel concetto che si definisce oggi come "One World - One Health", ovvero un mondo - una salute. In questo abbiamo un ruolo importante, e la riduzione dei farmaci, in particolare degli antibiotici, deve essere uno dei nostri principali obiettivi. Chiediamoci davvero se i nostri allevamenti potrebbero "ospitare" i nostri consumatori. Inviteremmo i nostri consumatori a visitare gli allevamenti? L'immagine che darebbero è davvero quella che noi vorremmo? Molti allevamenti, ne sono sicuro, sarebbero pronti ad ospitare, e molti stanno lavorando in questa direzione. Ma è una strada che si deve percorrere insieme. Insieme a tutta la filiera. Non può terminare alla stalla, anche il caseificio, l'industria di trasformazione deve fare la sua parte. Deve avere anche lei una impronta migliore, un'attenzione alla sostenibilità ambientale e non solo. Deve, per esempio, organizzarsi per ridurre l'impatto energetico e lo spreco dell'acqua.

Su tutti questi aspetti si sta lavorando molto in Europa e nel mondo.

Alcuni paesi hanno già calcolato l'impatto del settore lattiero caseario sull'ambiente. Sono calcoli non semplici, ma dovremmo farlo anche noi in Italia per avere un punto di partenza da cui iniziare un percorso virtuoso.

Lo dobbiamo a noi stessi, ai nostri consumatori. 